\title{
Electrochemical sensor for the detection of multiple reactive oxygen and nitrogen species from ageing central nervous system homogenates
}

\author{
A. Fagan-Murphy, L. Hachoumi, M.S. Yeoman and B.A. Patel
}

School of Pharmacy and Biomolecular Sciences, University of Brighton, Brighton, UK

Corresponding author: $\underline{\text { b.a.patel@brighton.ac.uk }}$

Reactive oxygen and nitrogen species (ROS/RNS) have been widely implicated in the ageing process and various approaches exist for monitoring these species in biological tissues. These approaches at present are limited to monitoring either a single pro-oxidant species or total pro-oxidant levels and therefore provide limited insight into the range of pro-oxidant species and their relative proportions in the ageing process. We have utilised a sensor that allows us to simultaneously monitor hydrogen peroxide, peroxynitrite, nitric oxide and nitrite. Using CNS homogenates from the pond snail, Lymnaea, we were able to show that levels of these ROS/RNS increased between young and old CNS homogenates and were different in various aged CNS regions.

The free radical theory of ageing is one of the most prominent theories to explain the ageing process (Harman, 1956). This theory postulates that ageing and age-related diseases arise as a result of cumulative changes and/or damage to cells and tissues inflicted by reactive species. Under normal physiological conditions, redox homeostasis establishes a dynamic balance between pro-oxidants and antioxidants whereby the production of reactive species are scavenged by antioxidants to minimise damage. However, with increasing age, studies have shown that the pro-oxidantantioxidant balance is compromised with a shift to the former resulting in an increase in reactive species. This imbalance induces oxidative stress, a state where endogenous antioxidant defense systems are overwhelmed leading to oxidative damage. More recent advances of this theory have shown that while, in laboratory reared animals, oxidative damage does not appear to affect lifespan it certainly increases oxidative damage and can contribute to age-related pathology (Salmon et 
al., 2010). Others have suggested that ageing represents a decline in the plasticity of pathways that have evolved to enable cells, tissue and organism to survive periods of environmental stress and increase fitness early in life (Jones, 2015). What all these theories have in common is that age is associated with an increase in the production of reactive species and being able to monitor these species simultaneously in realtime will be vital to furthering our knowledge of the ageing process and age-related pathology. There are various reactive species formed in biological environments, with a range of chemical reactivities which in turn can cause different levels of cellular damage. Little is however known about how the levels of different reactive oxygen and nitrogen species (ROS/RNS) vary with time in cellular environments during ageing

Determination of ROS/RNS species is a highly challenging analytical problem due to the low concentrations and high chemical reactivity of these species. Various methods have been developed in an attempt to measure ROS/RNS, of which fluorescence and chemiluminescence measurements have been most suitable for biological measurements (Kielland et al., 2009; Wang et al., 2013). However, these approaches have limitations due to their lack of selectivity for specific ROS/RNS. Electroanalytical approaches offer similar sensitivity, spatial and temporal resolution as these imaging based approaches, but, importantly, are able to simultaneously detect multiple identified ROS/RNS species in a single measurement (Amatore et al., 2008; Amatore et al., 2010).

Within this manuscript we demonstrate the fabrication and utilisation of a simple electrochemical sensor that offers the ability to simultaneously monitor the production of four ROS/RNS species. These are hydrogen peroxide $\left(\mathrm{H}_{2} \mathrm{O}_{2}\right)$, peroxynitrite (ONOO), nitric oxide (NO*) and nitrite $\left(\mathrm{NO}_{2}^{-}\right)$. The developed sensor was fabricated using multiwall-carbon nanotube (MWCNT) composite electrodes and modified by coating it with platinum black (Supplementary figure 1) using a previously published technology to enable the voltage-dependent detection of specific ROS/RNS (Amatore et al., 2008; Amatore et al., 2010). The sensors have been characterised for the detection of the four ROS/RNS in biological samples (see supplementary information for details on fabrication and characterisation of the sensors). To assess the sensor, levels of ROS/RNS were monitored from homogenates of young and old central nervous system (CNS) dissected from the pond snail Lymnaea stagnalis. As well as examining changes in the whole CNS, levels of ROS/RNS were also monitored in identified CNS ganglia to observe if the age-related changes in ROS/RNS were region specific. 
Supplementary figure 1 shows the reproducible production of the Pt-black MWCNT composite electrodes, where there was $\leq 5 \%$ standard deviation in the current response between 20 electrodes. To assess the Pt-black MWCNT composite electrodes, voltammograms were obtained for $\mathrm{H}_{2} \mathrm{O}_{2}, \mathrm{ONOO}^{-}, \mathrm{NO}^{*}$ and $\mathrm{NO}_{2}^{-}$(Figure 1A). The normalised current responses show the potential required to detect the various $\mathrm{ROS} / \mathrm{RNS}$, with $\mathrm{NO}_{2}{ }^{-}$requiring the highest over-potential for oxidation. Unlike other analytical approaches, the greater the potential applied the more species can be monitored (i.e. when $0.85 \mathrm{~V}$ is applied, the recorded oxidation current will be due to the oxidation of $\mathrm{H}_{2} \mathrm{O}_{2}, \mathrm{ONOO}^{-}, \mathrm{NO}^{*}$ and $\mathrm{NO}_{2}^{-}$). Therefore, to obtain selective detection of the various species, four voltages $(0.3,0.45,0.62$ and $0.85 \mathrm{~V})$ are applied on the sensor during a single recording. The current at these four voltages can be interpreted using equations 1-4 to establish the current specific for each ROS/RNS, which in turn can be converted to the quantity of each ROS/RNS using calibration curves.

$$
\begin{aligned}
& I^{0.85 \mathrm{~V}}=I_{\mathrm{H}_{2} \mathrm{O}_{2}}+I_{\mathrm{ONOO}^{-}}+I_{\mathrm{NO}}+I_{\mathrm{NO}_{2}^{-}} \\
& I^{0.62 \mathrm{~V}}=I_{\mathrm{H}_{2} \mathrm{O}_{2}}+I_{\mathrm{ONOO}^{-}}+I_{\mathrm{NO}} \\
& I^{0.45 \mathrm{~V}}=0.99 I_{\mathrm{H}_{2} \mathrm{O}_{2}}+0.92 I_{\mathrm{ONOO}^{-}} \\
& I^{0.3 \mathrm{~V}}=0.85 I_{\mathrm{H}_{2} \mathrm{O}_{2}}+0.27 I_{\mathrm{ONOO}^{-}}
\end{aligned}
$$

In Figure 1B, the suitability of the developed equations was assessed using solutions containing 100 nmoles of $\mathrm{H}_{2} \mathrm{O}_{2}, \mathrm{ONOO}^{-}, \mathrm{NO}^{\circ}$ and $\mathrm{NO}_{2}{ }^{-}$. The current detected at the four voltages in Figure 1B matches the voltammetric profile shown in Figure $1 \mathrm{~A}$ and therefore indicates that the electrode has the ability to selectively detect the four ROS/RNS. The ROS/RNS sensor has excellent linearity and limits of detection (Supplementary Table 1) and our findings are similar to those carried out on Pt-black microelectrodes and sensors placed in microchannels (Li et al., 2013).

Figure 2 shows responses from CNS homogenates from young and old Lymnaea CNSs. Measurements were made of the background saline and from old and young CNS homogenates. Clear increases in the current were observed at all the applied potentials (Figure 2A). Using the equations 1-4 and calibration responses, the quantity for $\mathrm{H}_{2} \mathrm{O}_{2}$, ONOO- (deprotonated form), $\mathrm{NO}^{*}$ and $\mathrm{NO}_{2}{ }^{-}$was obtained from both young and old CNS samples (Figure 2B). There was a significant increase in the amount of $\mathrm{H}_{2} \mathrm{O}_{2}(p<0.001, n=4)$ and $\mathrm{NO}_{2}(p<0.01, n=4)$ observed in old $C N S$ samples, 
when compared to young animals. Increases in $\mathrm{H}_{2} \mathrm{O}_{2}$ are predominantly observed as the stable by product following the formation of superoxide and its metabolism by superoxide dismutase. Although there were increases in ONOO- (deprotonated and protonated form) and NO these were not significant and showed much higher variability between aged CNS samples. Although the precise causes of this variability are unknown it may reflect changes in the animal's physiological state as NO' is an important neurotransmitter that has been shown to be activated during specific behaviours (Korneev et al., 1999). The observed variability may also be due to experimental factors, such as the exposure to atmospheric oxygen levels or due to the disruption of cellular integrity during homogenisation, which in turn may generate more free iron, which can enhance the amount of reactive species generated.

In order to explore whether the sensor was selective for the species detailed above in a complex biological sample, studies were carried out to investigate if reactive species scavengers could reduce levels of ROS/RNS in the aged animals. There was a significant reduction in $\mathrm{H}_{2} \mathrm{O}_{2}$ in the presence of tempol $(p<0.05, n=4)$. A reduction in NO* $(p<0.05)$ and $\mathrm{NO}_{2}{ }^{-}(p<0.001, n=4)$ was also observed in the presence of PTIO. The selective suppression of key ROS/RNS indicates that the sensor is capable of detecting these species in a complex biological sample.

Studies were carried out to understand if the age-related elevation in the amount of ROS/RNS species varied between the different regions of the old CNS (Figure 2D). The CNS was divided into the visceral parietal pedal (VPP) region and the cerebral buccal (CB) region. There was significantly more $\mathrm{H}_{2} \mathrm{O}_{2}$ produced in the VPP region than the CB region $(p<0.001, n=3)$. There was also a greater amount of $\mathrm{NO}_{2}{ }^{-}$in the $\mathrm{CB}$ region than the VPP region $(p<0.05, n=3)$. These results would indicate that there are region specific differences in which type of reactive species are generated, with more ROS being generated in the VPP region, with more RNS generated in the $\mathrm{CB}$ region.

Overall, our sensor has the ability to detect various chemical species that provide an indication of how pro-oxidant behaviour is altered with age. By having the ability to simultaneously monitor various pro-oxidants, the sensor can provide insight into which species are more prominent and which may affect nervous system function.

\section{Acknowledgements}

AFM, MSY and BAP would like to thank BBSRC TRDF for funding. 


\section{References}

Amatore, C., Arbault, S., Bouton, C., Drapier, J.C., Ghandour, H., Koh, A.C., 2008. Real-time amperometric analysis of reactive oxygen and nitrogen species released by single immunostimulated macrophages. Chembiochem 9, 1472-1480.

Amatore, C., Arbault, S., Koh, A.C., 2010. Simultaneous detection of reactive oxygen and nitrogen species released by a single macrophage by triple potential-step chronoamperometry. Anal Chem 82, 1411-1419.

Harman, D., 1956. Aging: A Theory Based on Free Radical and Radiation Chemistry. Journal of Gerontology 11, 298-300.

Kielland, A., Blom, T., Nandakumar, K.S., Holmdahl, R., Blomhoff, R., Carlsen, H., 2009. In vivo imaging of reactive oxygen and nitrogen species in inflammation using the luminescent probe L-012. Free Radical Biology and Medicine 47, 760-766.

Korneev, S.A., Park, J.-H., O'Shea, M., 1999. Neuronal Expression of Neural Nitric Oxide Synthase (nNOS) Protein Is Suppressed by an Antisense RNA Transcribed from an NOS Pseudogene. The Journal of Neuroscience 19, 7711-7720.

Li, Y., Sella, C., Lemaître, F., Guille Collignon, M., Thouin, L., Amatore, C., 2013. Highly Sensitive Platinum-Black Coated Platinum Electrodes for Electrochemical Detection of Hydrogen Peroxide and Nitrite in Microchannel. Electroanalysis 25, 895902.

Salmon, A.B., Richardson, A., Pérez, V.I., 2010. Update on the oxidative stress theory of aging: Does oxidative stress play a role in aging or healthy aging? Free radical biology \& medicine 48, 642.

Wang, X., Fang, H., Huang, Z., Shang, W., Hou, T., Cheng, A., Cheng, H., 2013. Imaging ROS signaling in cells and animals. Journal of Molecular Medicine (Berlin, Germany) 91, 917-927. 
Figure 1. Detection of ROS/RNS. (A) normalised voltammograms obtained at Pt-black MWCNT composite electrodes in 100 nmoles of $\mathrm{H}_{2} \mathrm{O}_{2}$, ONOO', $\mathrm{NO}^{-}$and $\mathrm{NO}_{2}{ }^{-}$at varying potentials relative to a $\mathrm{Ag} \mid \mathrm{AgCl}$ reference electrode. The dashed line indicates the potentials that were utilised for detection of the four different ROS/RNS. In order to obtain the current for each of the species, equation 1-4 were applied. (B) Shows response at the chosen potentials from (A) for 100 nmoles of $\mathrm{H}_{2} \mathrm{O}_{2}$, ONOO', NO ${ }^{*}$ and $\mathrm{NO}_{2}{ }^{-}$. Measurements were carried out in stirred solution, where stable responses were observed that mapped well to the profile obtained in (A). For all measurements the $\mathrm{pH}$ was 7.4 for $\mathrm{H}_{2} \mathrm{O}_{2}, \mathrm{NO}^{*}$ and $\mathrm{NO}_{2}{ }^{-}$and $\mathrm{pH} 11.5$ was utilised for recordings of $\mathrm{ONOO}^{-}$.

Figure 2. Detection of ROS/RNS levels from CNS homogenates. (A) multiple-step amperometry waveform utilised, where responses of the HEPES buffer (control), homogenate of young and old CNS at $0.3,0.45,0.62$ and $0.85 \mathrm{~V}$ vs $\mathrm{Ag} \mid \mathrm{AgCl}$ for $30 \mathrm{~s}$ was obtained. A large charging current was obtained when each potential was applied to the sensor. The current was monitored at each potential at $t=29 \mathrm{~s}$ within the recording and determination of $\mathrm{H}_{2} \mathrm{O}_{2}, \mathrm{ONOO}^{-}, \mathrm{NO}^{-}$and $\mathrm{NO}_{2}^{-}$were obtained using equations 1-4. (B) Responses of the levels of $\mathrm{H}_{2} \mathrm{O}_{2}, \mathrm{ONOO}^{-}, \mathrm{NO}^{-}$and $\mathrm{NO}_{2}^{-}$in whole CNS from young and old Lymnaea stagnalis. (C) Response of $\mathrm{H}_{2} \mathrm{O}_{2}, \mathrm{ONOO}^{-}, \mathrm{NO}^{\circ}$ and $\mathrm{NO}_{2}^{-}$in whole CNS from old Lymnaea stagnalis in the presence of reactive species scavengers, $1 \mathrm{mM}$ PTIO and $1 \mathrm{mM}$ tempol. (D) Responses from old CNS regions, where the VPP is the visceral parietal and pedal region and the CB is the cerebral buccal region. All data shown as mean \pm St.dev. and $n=3-4$. 
FIGURE 1

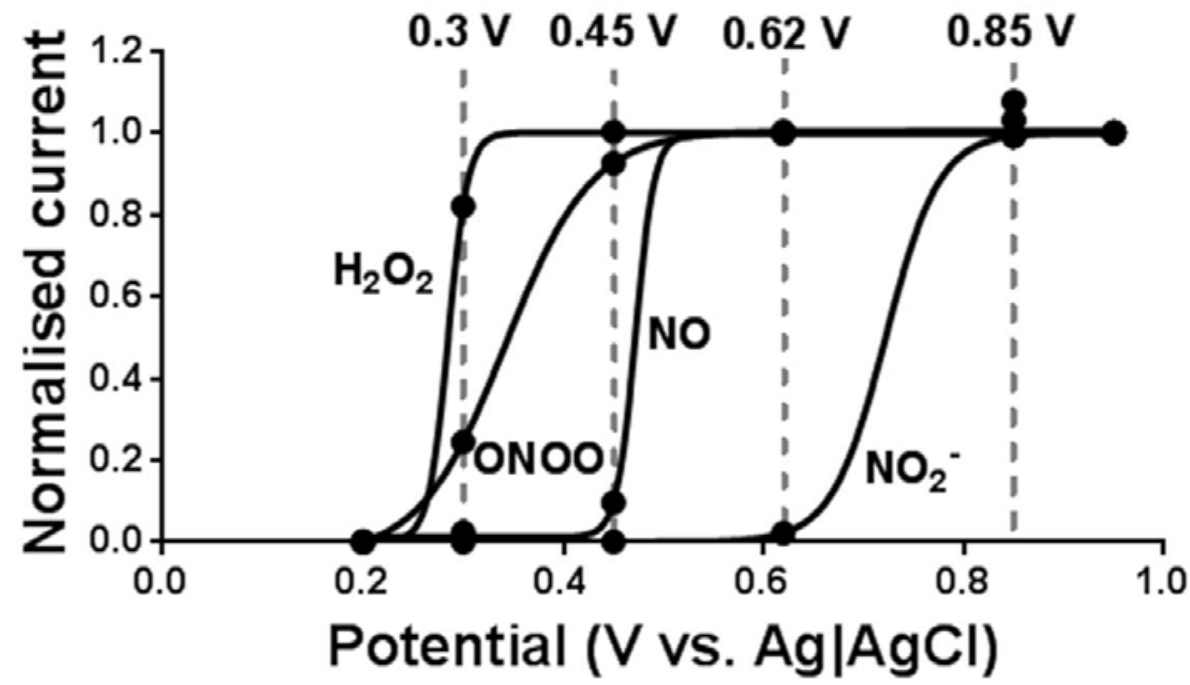

B

$\mathrm{H}_{2} \mathrm{O}_{2}$
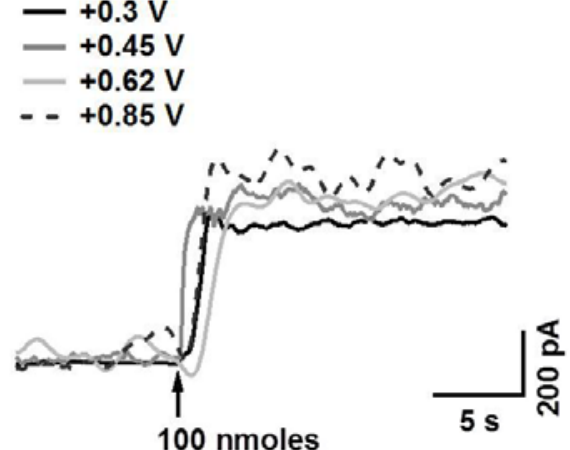

NO
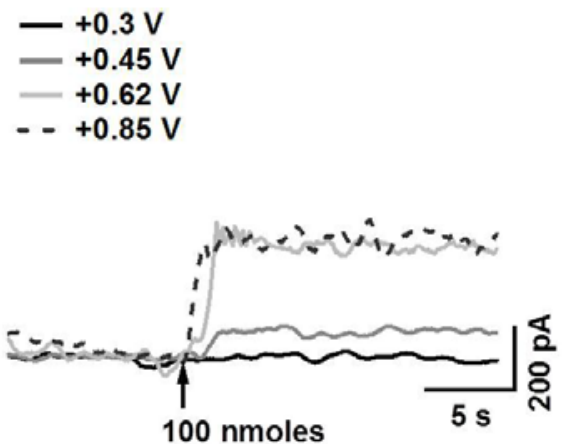

\section{ONOO-}

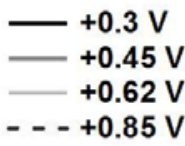

$\mathrm{NO}_{2}{ }^{-}$

- $+0.3 \mathrm{~V}$

$-+0.45 \mathrm{~V}$

$-+0.62 \mathrm{~V}$

- + $+0.85 \mathrm{~V}$

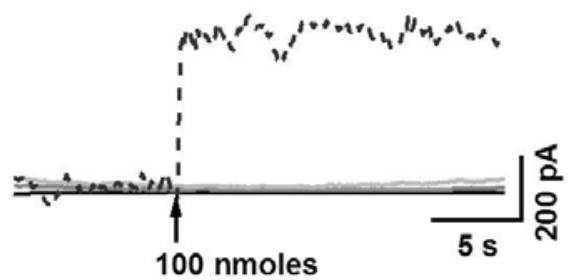


FIGURE 2

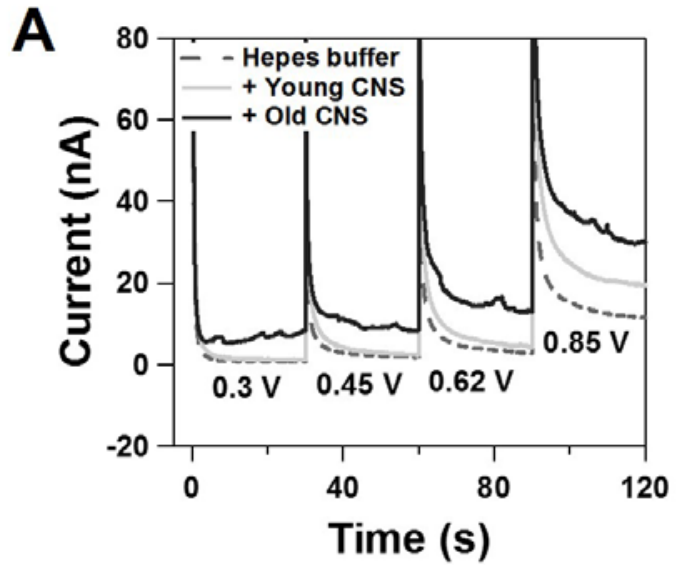

B

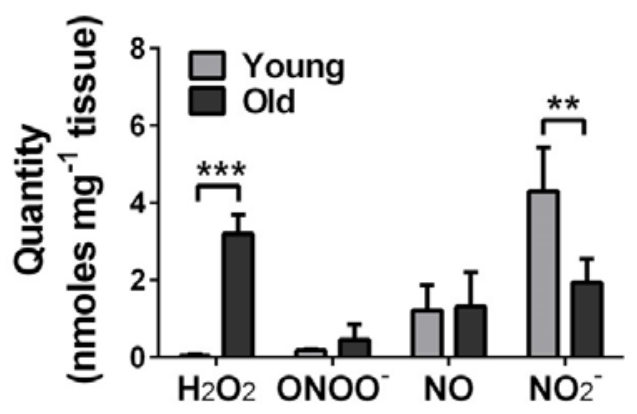

C
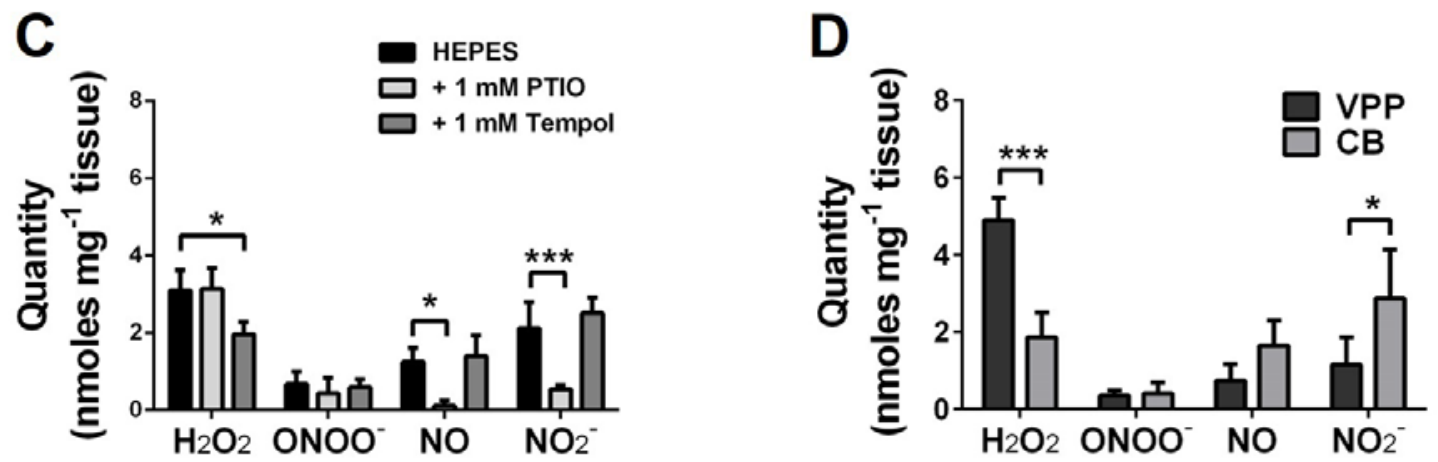